\title{
SOME ACHIEVEMENTS OF NEVANLINNA THEORY
}

\author{
W. K. HAYMAN
}

We have come together at this conference to honour Rolf Nevanlinna. Hilbert once said to him: "You have made a hole in the wall of Mathematics. Other mathematicians will fill it." If the hole means that many new problems were opened up, then this is indeed the case, and I am certain that Nevanlinna theory will continue to solve problems as it has done in the last 50 years. Today I would like to give an account of a few questions which have been wholly or partially solved by means of the ideas in this theory.

1. Deficient and asymptotic values. When Nevanlinna first came to the University of Helsinki, Iversen was an assistant there. He had proved a famous theorem [17]. If $f$ is a non-constant entire function, then

$$
f \rightarrow \infty \text { along some path } \Gamma_{\infty} .
$$

More generally if $f$ is meromorphic transcendental and assumes the value $a$ only a finite number of times then $a$ is an asymptotic value, i.e.

$$
f \rightarrow a \text { as } z \rightarrow \infty \text { along some path } \Gamma_{a} .
$$

The path $\Gamma_{a}$ is called an asymptotic path.

It is natural to ask whether a corresponding result holds if the equation $f=a$ has sufficiently few roots and Nevanlinna theory enables us to define what we mean by that. Nevanlinna conjectured that deficient values might be asymptotic, i.e. $\delta(a, f)>0$ implies (1). If

$$
T(r, f)=O(\log r)^{2}
$$

this is indeed the case, as was proved by Anderson and Clunie [3]. If $f$ has finite order $\varrho$, and $1-\delta(\infty, f)$ and $2-\Sigma \delta(a, f)$ are sufficiently small depending on $\varrho$, the conjecture was proved by Edrei and Fuchs [8], sharpening an earlier result of Pfluger [21].

For general functions the result is false if $f$ grows more rapidly that is implied by (2). However [12] if

$$
T(r, f)-\frac{1}{2} r^{1 / 2} \int_{r}^{\infty} \frac{N(t, a) d t}{t^{3 / 2}} \rightarrow+\infty,
$$


then $a$ is indeed an asymptotic value. This condition is satisfied in particular if $\delta(a, f)>0$ and $f$ has smooth slow growth, e.g.

$$
\frac{T(2 r)}{T(r)} \rightarrow 1,
$$

a condition implied by (2). Further Anderson [2] has shown in this case that the path $\Gamma_{a}$ can be chosen to be almost straight. It can be chosen to be a straight line, or more generally almost every straight line through the origin if the stronger condition (2) holds [3] but not if just (4) is satisfied [12].

For functions of rapid growth and in particular infinite order we cannot improve substantially the condition (3). Given any sequence of positive numbers $r_{n}$ tending to $\infty$ and such that

$$
\sum r_{n}^{-3 / 2}=\infty,
$$

which is equivalent to $\int_{0}^{\infty} r^{-3 / 2} N(r) d r=\infty$ for the counting function, there exists an entire function $f$, of arbitrarily rapid growth as measured by $T(r, f)$, which has no zeros at points other than the $r_{n}$ but for which zero is not an asymptotic value [13]. On the other hand (3) shows that if

$$
\int \frac{N(t, a) d t}{t^{3 / 2}}<\infty
$$

and $\varliminf_{r \rightarrow \infty} r^{-1 / 2} T(r, f)>0$, then $a$ is asymptotic.

2. Size of $m(r, a)$ for varying $a$. According to the first fundamental Theorem we have for $f$ meromorphic in $|z|<R$

$$
m(r, a)+N(r, a)=T(r)+O(1) \quad \text { as } \quad r \rightarrow R .
$$

The second fundamental Theorem shows that in this relation the term $m(r, a)$ is in general small for most values of $a$. It is natural to ask "how small and for how many $a$ ?".

In the first instance it follows from results of Nevanlinna and Frostman [20, pp. 171, 172] together with Choquet's theory of capacitability [14, Chapter 5], that

$$
m(r, a) \rightarrow \infty
$$

can only hold for a set of capacity zero. Drasin and Weitsman [7] have shown that this is sharp. Given any set $A$ of capacity zero and $\varrho>1 / 2$, there exists an entire function of order $\varrho$, such that (5) holds for every $a$ in $A$. If $\varrho=1 / 2$, then $A$ must be contained in an $F_{\sigma}$ set of capacity zero.

At the other end of the scale we may ask if $m(r, a)$ can sometimes be large for every $a$ and if so how large. Here the answer is given by an old Theorem of Littlewood [18]

$$
\varlimsup_{r \rightarrow R} \frac{m(r, a)}{\log T(r)} \leqq \frac{1}{2}
$$


outside a set of areal measure zero. This was generalized by Ahlfors [1] who proved that

$$
\varlimsup \frac{m(r, a)}{\log T(r)} \leqq \frac{1}{\delta}
$$

outside a set of $\delta$-dimensional measure zero.

Nevanlinna [20, p. 201] showed that if $E$ is a closed set of capacity zero and $f(z)$ maps $|z|<1$ onto the universal covering surface over the complement of $E$ then $f$ has unbounded characteristic. Since $f$ does not assume any value of $E$, we have for $a \in F$

$$
m(r, a)=T(r)+O(1) \quad \text { as } \quad r \rightarrow 1, \quad N(r, a)=0 .
$$

Nevanlinna [20, p. 263] showed that outside a set of capacity zero $m(r, a)$ must be small compared with $T(r)$. More precisely if $\eta>1 / 2$, then if $f$ has unbounded characteristic in $|z|<R$

$$
m(r, a)=O\left\{T(r)^{\eta}\right\}, \quad \text { as } r \rightarrow R
$$

outside a set of capacity zero.

Ahlfors [1] had shown earlier that if $0<\eta \leqq 1 / 2$ then (8) holds outside a set $E(\eta)$, which can be enclosed in a sequence of disks of radii $\varrho_{n}$, for which

$$
\sum \frac{1}{\left(\log \frac{1}{\varrho_{n}}\right)^{(1-\eta) / \eta}}
$$

can be made arbitrarily small. We may say that the logarithmic dimension of the exceptional set is at most $(1-\eta) / \eta$.

All these results are essentially best possible. One can construct counter examples of functions of unbounded characteristic in the unit disk such that

$$
\varlimsup \frac{m(r, a)}{\log T(r)} \geqq \frac{1}{2}
$$

for every $a$. Given a set $E_{\beta}$ of Hausdorff dimension $\beta$, we can make

$$
\varlimsup \frac{m(r, a)}{\log T(r)} \geqq \frac{1}{\beta}
$$

on every point of $E_{\beta}$. Given a set $F_{\eta}$ of logarithmic dimension $(1-\eta) / \eta$, where $\eta \leqq 1 / 2$, we can find $f$ such that

$$
\varlimsup \frac{\log m(r, a)}{\log T(r)} \geqq \eta
$$

for every $a \in F_{\eta}$. In particular even for $\eta=1 / 2$, we can choose $F_{\eta}$ to have positive capacity. Thus Nevanlinna's Theorem is no longer true for $\eta<1 / 2$, and here Ahlfors' Theorem gives the sharp result. 
It follows from Nevanlinna's theorem that

$$
\varlimsup \frac{m(r, a)}{T(r)}>0
$$

can hold at most on a set of capacity zero. For functions of infinite order this is also sharp. If $E$ is an $F_{\sigma}$ set of capacity zero, there exists an entire function of infinite order such that

$$
\varlimsup \frac{m(r, a)}{T(r)}=1
$$

for every $a$ in $E$. However for functions of finite order Hyllengren [16] has shown that the exceptional set is much smaller than this. If the enclosing circles are again $\left|z-z_{n}\right|<\varrho_{n}$, we can make $\sum(\log \log (1 / \varrho))^{-(1+\varepsilon)}$ small, when $\varepsilon>0$, but not in general when $\varepsilon<0$.

3. Picard values of derivatives. Suppose that $f$ is transcendental meromorphic it the plane. What can we say about the values assumed by the derivatives $f^{(l)}$ of $f$ ?

In view of Picard's theorem $f$ can leave out at most 2 finite values. However Nevanlinna's theory tells us [11] that $f^{(l)}$ can leave out at most one such value. For every pole of $f^{(l)}$ has multiplicity at least $l+1 \geqq 2$, so that the sum of the other deficiencies is at most $1+1 /(l+1) \leqq 3 / 2$.

If $f$ already has a finite Picard value, then the exceptional value of $f^{(l)}$ can only be zero. An example is given by $f=e^{z}+a$. None of the derivatives assume the value zero. If we set $f(z)=e^{\int g(z) d z}$, where $g(z)$ is entire then $f \neq 0, f^{\prime} \neq 0$. But for higher derivatives no such examples can exist. Frank and the Hennekempers [9, 10] proved a conjecture of mine [11] that if $f$ is meromorphic and $f \neq 0, f^{(l)} \neq 0$ for some $l \geqq 2$, then either $f=e^{a z+b}$ or $f=(a z+b)^{-n}$, for constant $a, b$ and a positive integer $n$. This settled finally old problems going back to Pólya and Csillag [6]. Nevanlinna's theory was an essential tool in this work.

Problems of Picard values of various polynomials in $f$ and its derivatives can also be attacked. I cite an example. If $f$ is meromorphic transcendental then $f^{\prime} f^{n}$ assumes every value except zero infinitely often if $n \geqq 2[11,19]$. If $f$ is entire the result is true for $n \geqq 1$ [5]. Also $f^{\prime}-a f^{n}$ for $a \neq 0$ assumes every value if $n \geqq 5$ [11], and the value zero also for $n=4$, but if $n=3$ or 4 and $c \neq 0$, there exist functions for which $f^{\prime}-a f^{n} \neq c$ [19]. It is not known if $f^{\prime} f$ assumes all values except zero or whether $f^{\prime}-f^{3}$ necessarily has zeros, when $f$ is meromorphic.

4. Iteration. Suppose that $f$ is non linear entire and define $f_{n}$ inductively by $f_{1}=f, f_{n+1}(z)=f\left\{f_{n}(z)\right\}$. Then the $f_{n}$ are called the iterates of $f$. If $f_{n}(\zeta)=\zeta$, but $f_{p}(\zeta) \neq \zeta$ for $1<p<n$ we say that $\zeta$ is a fix point of exact order $n$. It was shown by Baker [4] using Nevanlinna theory, that every $f$ has fix-points of all exact orders with at most one exception. The function $f(z)=e^{z}+z$ shows that there need be no fix points of order 1 . The polynomial $f(z)=z^{2}-z$ has no fix points of exact 
order 2. However the question of whether a transcendental entire function can have an exceptional order other than 1 or a polynomial an exceptional order other than 2 , remains open.

5. Angular value distribution. Nevanlinna theory shows that for meromorphic functions in the plane the roots of equations $f=a$ are distributed with great regularity. All values are taken roughly equally often, except possibly two, which may be taken less often. For functions meromorphic in an angle the results are much less precise but with the aid of modified Nevanlinna theory we can say a good deal in this case also.

Let $\alpha<\arg z<\beta$ be an angle $S$. Let $n(r, \alpha, \beta, a)$ be the number of roots of $f=a$ in $\{S \cap|z|<r\}$, and define

$$
\varrho_{0}(\alpha, \beta, a)=\varlimsup_{r \rightarrow \infty} \frac{\log n(r, a)}{\log r} .
$$

The order $\varrho(\alpha, \beta, a)$ is defined suitably as

$$
\varrho(S, a)=\varrho(\alpha, \beta, a)=\lim _{\varepsilon \rightarrow 0^{+}} \varrho_{0}(\alpha+\varepsilon, \beta-\varepsilon, a) .
$$

We can think of this as the inner order. Then Valiron [24, p. 31] proved essentially the following

Theorem. There exists $\varrho=\varrho(S)$, such that

$$
\varrho(S, a)=\varrho
$$

except for at most two values a for which $\varrho(S, a)<\varrho$ and a set $V$ of linear measure zero for which $\varrho(S, a)>\varrho$.

The exceptional set $V$ can in fact exist. It is even smaller than Hyllengren's [16] sets and can be enclosed in disks $\left|z-z_{n}\right|<\varrho_{n}$ for which

$$
\sum\left(\log ^{+} \log ^{+} \log +\frac{1}{\varrho}\right)^{-1-\varepsilon}
$$

can be made as small as we please and a much more precise characterization can be given, using Hyllengren's concept of span.

6. Extensions. The theory of Nevanlinna has many other applications. Some of these have been considered by other speakers. I would like to mention the relation between the growth of $f$ and $f^{\prime}$, where distinguished work has recently been done by Toppila [22, 23], and entire functions which, together with some derivatives, have only real zeros (Hellerstein and Williamson [15]) and the whole idea of functions of bounded characteristic [20, Chapter 7], a concept which has proved of major importance in many areas. Perhaps it is worth saying a word about subharmonic functions in $R^{m}, m \geqq 3$. 
If $u(x)$ is such a function it can be expressed on any compact set $E$ as the sum of potential $p(x)$ and a function $h(x)$ harmonic in the interior of $E$. Here

$$
p(x)=\int_{E}|x-\xi|^{1-m} d \mu(\xi)
$$

and the positive measure $\mu$ is called the Riesz mass of $\mu$. We denote by $n(t)$ the Riesz mass of the ball $|x| \leqq t$ and write

$$
N(r)=(m-2) \int_{0}^{r} \frac{n(t) d t}{t^{m-1}} .
$$

If $u^{+}(x)=\max (u, 0), u^{-}(x)=\max (-u, 0)$, we write

$$
\begin{aligned}
T(r, u) & =\frac{1}{c_{m} r^{m-1}} \int_{|x|=r} u^{+}(x) d \sigma(x) \\
m(r, u) & =\frac{1}{c_{m} r^{m-1}} \int_{|x|=r} u^{-}(x) d \sigma(x) .
\end{aligned}
$$

Here $\sigma(x)$ is superficial measure on $|x|=r$ and $c_{m}$ is the measure of the unit sphere in $R^{m}$. We then obtain the first fundamental Theorem in the form

$$
T(r, u)=m(r, u)+N(r, u)+u(0)
$$

provided that $u(0)>-\infty$. The function $T(r, u)$ gives a very good description of the growth of the subharmonic function $u(x)$ and its asymptotic behaviour as $x \rightarrow \infty$ and the analogues of the results of Weierstrass and Hadamard for entire functions in the plane can be obtained without too much difficulty. Functions of bounded characteristic in $R^{m}$ are precisely those functions which are bounded above, but these functions need not be constant [14, Chapters 3 and 4]. The analogues of meromorphic functions are the so called $\delta$-subharmonic functions, which are locally the differences of two subharmonic functions. Since any twice continuously differentiable function is $\delta$-subharmonic further restrictions must be made in general to obtain significant theorems.

I hope that this far from exhaustive survey has given some impression of the power and beauty of Nevanlinna theory.

\section{References}

[1] Ahlfors, L.: Ein Satz von Henri Cartan und seine Anwendung auf die Theorie der meromorphen Funktionen. - Soc. Sci. Fenn. Comment. Phys.-Math. 16, 1931, 1-19.

[2] Anderson, J. M.: Asymptotic values of meromorphic functions of smooth growth. - Glasgow Math. J. 20, 1979, 155-162.

[3] Anderson, J. M., and J. G. Clunie: Slowly growing meromorphic functions. - Comment. Math. Helv. 40, 1966, 267-280.

[4] Baker, I. N.: The existence of fixpoints of entire functions. - Math. Z. 73, 1960, 280-284. 
[5] Clunie, J. G.: On a result of Hayman. - J. London Math. Soc. 42, 1967, 389-392.

[6] CsillaG, P.: Über ganze Funktionen, welche drei nicht verschwindende Ableitungen besitzen. Math. Ann. 110, 1935, 745-752.

[7] Drasin, D., and A. Weitsman: The growth of the Nevanlinna proximity function and the logarithmic potential. - Indiana Univ. Math. J. 20, 1971, 699-715.

[8] Edrei, A., and W. H. J. Fuchs: Valeurs déficientes et valeurs asymptotiques des fonctions méromorphes. - Comment. Math. Helv. 33, 1959, $292-328$.

[9] Frank, G.: Eine Vermutung von Hayman über Nullstellen meromorpher Funktionen. - Math. Z. 149, 1976, 29-36.

[10] Frank, G., W. Hennekemper, and G. Polloczek: Über die Nullstellen meromorpher Funktionen und deren Ableitungen. - Math. Ann. 225, 1977, 145-154.

[11] Hayman, W. K.: Picard values of meromorphic functions and their derivatives. - Ann. of Math. 70, 1959, 9-42.

[12] HaYman, W. K.: On Iversen's theorem for meromorphic functions with few poles. - Acta Math. 141, 1978, 115-145.

[13] Hayman, W. K.: On a meromorphic function having few poles, but not tending to infinity along a path. - Ann. Polon. Math. 39, 1981, 84-91.

[14] Hayman, W. K., and P. B. Kennedy: Subharmonic functions (1). - Academic Press, New York-London, 1976.

[15] Hellerstein, S., and J. Williamson: Derivatives of entire functions and a question of Pólya. Trans. Amer. Math. Soc. 227, 1977, 227-249 and 234, 1977, 497-503.

[16] Hyllengren, A.: Valiron deficient values for meromorphic functions in the plane. - Acta Math. 124, 1970, 1-8.

[17] IVERSEN, F.: Sur quelques propriétés des fonctions monogènes au voisinage d'un point singulier. - Översikt av Finska vetenskaps-societetens förhandlingar 58. A. 25, Helsingfors, 1915-1916.

[18] Littrewood, J. E.: Mathematical Notes (11): On exceptíonal values of power series. - J. London Math. Soc. 5, 1930, 179-182.

[19] Mues, E.: Über ein Problem von Hayman. - Math. Z. 164:3, 1979, 239-259.

[20] Nevanlinna, R.: Eindeutige analytische Funktionen. - Die Grundlehren der mathematischen Wissenschaften 46, Verlag von Julius Springer, Berlin, 1936.

[21] Pfluger, A.: Zur Defektrelation ganzer Funktionen endlicher Ordnung. - Comment. Math. Helv. 19, 1946, 91-104.

[22] ToppIla, S.: On Nevanlinna's characteristic function of entire functions and their derivatives. Ann. Acad. Sci. Fenn. Ser A I Math 3, 1977, 131-134.

[23] Toppila, S.: On the characteristic of meromorphic functions and their derivatives. - J. London Math. Soc. (2) (to appear).

[24] Valiron, G.: Directions de Borel des fonctions méromorphes. - Mémor. Sci. Math. 89, 1938.

\author{
Imperial College \\ Department of Mathematics \\ London SW7 2BZ \\ England
}

Received 6 March 1981 